Revista Med 26(2) 2018

\title{
EVALUACIÓN DE LA GESTIÓN DE RECETAS EN UN HOSPITAL DISTRITAL DEL DEPARTAMENTO CENTRAL
}

\author{
MARÍA FERNANDA CABALLERO ${ }^{1}$, GLORIA ORREGO², MARÍA GLORIA DOMENEH ${ }^{3}$, \\ PATRICIA ACOSTA ${ }^{4}$, ZULLY VERA ${ }^{5}$, GLADYS BEATRIZ LUGO ${ }^{6}$, GLADYS MABEL MAIDANA ${ }^{7}$ \\ ${ }^{1}$ Química Farmacéutica Hospital Distrital de Lambarè, Paraguay. \\ ${ }^{2}$ Química Farmacéutica. Hospital Distrital de Lambarè, Paraguay. \\ ${ }^{3}$ Docente Investigadora, Departamento de Farmacia, Facultad de Ciencias Químicas, \\ Universidad Nacional de Asunción, Paraguay. \\ ${ }^{4}$ Facultad de Ciencias Químicas, Universidad Nacional de Asunción, Paraguay. \\ ${ }^{5}$ Docente Investigadora, Departamento de Farmacia, Facultad de Ciencias Químicas, \\ Universidad Nacional de Asunción, Paraguay. \\ ${ }^{6}$ Docente Investigadora. Departamento de Farmacia, Facultad de Ciencias Químicas, \\ Universidad Nacional de Asunción, Paraguay. \\ ${ }^{7}$ Docente Investigadora, Departamento de Farmacia, Facultad de Ciencias \\ Químicas, Universidad Nacional de Asunción, Paraguay. \\ Correspondencia: Gladys Mabel Maidana \\ glamaida73@gmail.com
}

Recibido: 9 de julio de 2018 Aceptado: 31 de agosto

\section{Resumen}

Introducción: El error de medicación es cualquier evento evitable que puede estar relacionado con la práctica profesional, derivado de acciones desacertadas, que pueden afectar la salud y que van desde el momento de la prescripción hasta el cumplimiento de la orden médica. Los errores de prescripción son relevantes, ya que, si no son detectados a tiempo, pueden significar daño o muerte al paciente. Objetivo: Evaluar las recetas de pacientes ambulatorios en la farmacia interna de un hospital distrital entre enero y abril de 2017. Metodología: estudio observacional descriptivo, retrospectivo con muestreo aleatorio simple. La recopilación de datos se realizó mediante la revisión de recetas prescritas a pacientes que acuden al consultorio externo, teniendo en cuenta los indicadores de la Organización Mundial de la Salud (OMS) para el uso de fármacos. Los datos fueron registrados en una planilla de Excel junto con los parámetros por ser evaluados. Resultados: De las 3376 prescripciones analizadas, 1199 (35,5\%) cumplían con todos los requisitos legales, 2507 (74,3\%) estaban escritas con letra legible, 3117 (92,3\%) contaban con el diagnóstico del paciente, $788(53 \%)$ tenían especificada la dosis del medicamento por ser dispensado y $2672(79,1 \%)$ tenían aclarada la forma farmacéutica. Conclusión: Se debe trabajar en la detección, la prevención y el tratamiento de los errores de medicación mediante la integración de un equipo multidisciplinario, la estandarización de la prescripción médica y la implementación de la receta electrónica de manera que se facilite la prescripción de los medicamentos.

Palabras clave: prescripciones; errores de medicación; atención farmacéutica 


\title{
EVALUATION OF PRESCRIPTION MANAGEMENT IN A CENTRAL DEPARTMENT DISTRICT HOSPITAL
}

\begin{abstract}
Introduction: A medication error is any avoidable event that may be related to professional practice, derived from misguided actions, which may affect health and that range from the time of prescription to the fulfillment of the medical order. Prescription errors are relevant, since, if they are not detected in time, they can mean harm or death to the patient. Objective: To evaluate outpatient prescriptions in the internal pharmacy of a district hospital between January and April 2017. Methodology: descriptive, retrospective observational study with simple random sampling. Data collection was performed by reviewing prescriptions prescribed to patients who go to the outpatient clinic, taking into account the indicators of the World Health Organization (WHO) for the use of drugs. Data were recorded in an Excel spreadsheet along with the parameters to be evaluated. Results: Out of the 3376 prescriptions analyzed, 1199 (35.5\%) met all legal requirements, 2507 (74.3\%) were written in legible letters, 3117 (92.3\%) had the patient's diagnosis, 788 (53\%) had specified the dose of the drug to be dispensed and 2672 (79.1\%) had clarified the pharmaceutical form. Conclusion: Work should focus on detection, prevention and treatment of medication errors through the integration of a multidisciplinary team, standardization of medical prescription and implementation of electronic prescription in order to ease the medication prescription.
\end{abstract}

Keywords: prescriptions; medication errors; pharmaceutical care.

\section{AVALIAÇÃO DO GERENCIAMENTO DE RECEITAS NUM HOSPITAL DISTRITAL DO DEPARTAMENTO CENTRAL}

\section{Resumo}

Introdução: o erro de medicação é qualquer acontecimento evitável que pode estar relacionado com a prática profissional, derivado de ações desacertadas, que podem afetar a saúde e que vão desde o momento da prescrição até o cumprimento da ordem médica. Os erros de prescrição são relevantes, já que, se não são detectados a tempo, podem significar dano ou morte ao paciente. Objetivo: avaliar as receitas de pacientes de ambulatórios na farmácia interna de um hospital distrital entre janeiro e abril de 2017. Metodologia: estudo observacional descritivo, retrospectivo com amostragem aleatória simples. A recopilação de dados realizou-se mediante a revisão de receitas prescritas a pacientes que vão ao consultório externo, considerando os indicadores da Organização Mundial da Saúde (OMS) para o uso de fármacos. Os dados foram registrados numa planilha de Excel junto com os parâmetros a serem avaliados. Resultados: Das 3376 prescrições analisadas, 1199 (35,5\%) cumpriam com todos os requisitos legais, 2507 (74,3\%) estavam escritas com letra legivel, 3117 (92,3\%) contavam com o diagnóstico do paciente, 788 (53\%) tinham especificada a dose do medicamento a ser dispensado e $2672(79,1 \%)$ tinham esclarecido a forma farmacêutica. Conclusão: deve ser trabalhado na detecção, na prevenção e no tratamento dos erros de medicação mediante a integração de uma equipe multidisciplinar, a padronização da prescrição médica e a implementação da receita eletrônica de maneira que se facilite a prescrição dos medicamentos.

Palavras-chave: prescrições; erros de medicação; atendimento farmacêutico 


\section{Introducción}

Desde la publicación en 2000 del informe To err is human: building a safer health system, del Instituto de Medicina de los Estados Unidos, la seguridad del paciente dentro de los hospitales ha mejorado, debido a una serie de medidas legislativas y reglamentarias impulsadas por las autoridades, dada la importante repercusión asistencial y económica que tienen los daños prevenibles derivados de errores durante la asistencia sanitaria $(1,2)$.

Entre los incidentes derivados de la asistencia clínica, los más prevalentes son los errores asociados al uso de medicamentos, que pueden tener lugar en cualquier etapa del circuito de utilización (3).

Los errores de medicación son definidos como "cualquier incidente prevenible que puede causar daño al paciente o dar lugar a una utilización inapropiada de los medicamentos, cuando estos están bajo control de profesionales sanitarios o del paciente o consumidor" (4).

Entre ellos, están los errores en las recetas médicas que provienen de fallas en la escritura de la receta, que da lugar a una instrucción errónea acerca de una o más de las "características normales" de una receta (identidad de quien la recibe, identidad de la droga, formulación, dosis, vía, tiempo, frecuencia y duración de la administración) (5).

Se agregan, además, los errores de dispensación, que se definen como la discrepancia entre lo que indica una receta médica y el medicamento dispensado por la farmacia al paciente, o que distribuye a la sala, sobre la base de esta receta, que incluye la dispensación de un medicamento con inferior calidad farmacéutica o de información (6).

Si este error es detectado antes que los medicamentos lleguen al paciente, se denomina dispensing near miss. Para el presente estudio, se entenderá este último como un error en la preparación de las recetas, pesquisado antes de entregar los medicamentos al paciente (7).

Aunque las tasas de errores en la dispensación son generalmente bajas, las mejoras en los sistemas de distribución de farmacia siguen siendo importantes, porque estas dispensan volúmenes tan altos de medicamentos que incluso una baja tasa de error puede traducirse en un gran número de errores (8).
En pacientes ambulatorios, la incidencia de acontecimientos prevenibles por medicamentos es del 5,4\% (4). Algunos autores han determinado, además, que la tasa de error oscila entre el 3,5 y el 11,4\% de las dosis administradas, y que un paciente hospitalizado presenta 0,9 errores de medicación diarios $(4,9,10)$.

El $42 \%$ de los eventos adversos potencialmente mortales o graves se deben a errores en la prescripción, en que la tasa de error oscila entre el 6,3 y el $14,7 \%$ de errores en las recetas manuscritas (11-13).

Como no existe una estimación de la magnitud y las características de los errores de medicación en el hospital distrital, esta investigación tiene como principal objetivo evaluar las recetas de pacientes ambulatorios en la farmacia interna de un hospital distrital entre los meses de enero y abril de 2017, según los indicadores de la Organización Mundial de la Salud (OMS).

\section{Material y método}

Este es un estudio observacional descriptivo, retrospectivo, de muestreo aleatorio simple, sin reposición, realizado en junio 2017 en la farmacia interna de un hospital distrital del departamento central, establecimiento de mediana complejidad que es una institución pública, perteneciente a la red del Ministerio de Salud Pública y Bienestar Social, en la cual no se había realizado ninguna evaluación sobre la gestión de las recetas hasta la fecha.

Esta farmacia dispensa recetas a pacientes crónicos atendidos en los consultorios policlínicos de medicina interna, pacientes de morbilidad atendidos en policlínicos de otras especialidades (traumatología, pediatría, oftalmología, entre otros), de urgencia y también a pacientes que una vez finalizado su proceso de hospitalización son dados de alta con tratamiento farmacológico.

Se incluyeron las recetas de pacientes ambulatorios adultos y pediátricos, prescritas por médicos de los consultorios externos, en los meses de enero, febrero, marzo y abril de 2017. Durante este periodo fueron dispensadas 39012 recetas.

El cálculo de muestra para las recetas médicas consideró una prevalencia de errores del $4 \%$, basada en un estudio piloto de 40 recetas elegidas al azar y respaldada por lo publicado por Otero (4), con un nivel de confianza del 95 y del $0,6 \%$ de precisión, lo que determinó finalmente un $n=3376$. La selección de las recetas fue en forma aleato- 
ria, comenzando con un número al azar y, posteriormente, una de cada doce se seleccionaba para ser evaluada, hasta llegar a las 28 diarias necesarias para completar el número de recetas determinado para estudiar.

La recolección de los datos fue manual, mediante la revisión individualizada de cada receta, luego se procedió al llenado de indicadores por medir teniendo en cuenta los indicadores de la OMS del uso de fármacos, registrando en una planilla de Excel. La pauta para calcular errores en las recetas médicas quedó conformada por 13 ítems que representan posibles errores en las características de la receta. Se aplicó la pauta de chequeo de lunes a domingo, en forma aleatoria, a 28 recetas del total del día. Se analizaron los errores en las recetas médicas con sus respectivas frecuencias.

\section{Resultados}

Se evaluaron 3376 recetas médicas. El número de prescripciones por receta médica varía de uno a diez medicamentos, se encontraron 1290 (38\%) recetas con la prescripción de dos medicamentos. La frecuencia de cantidad de medicamento se detalla en la tabla 1.

Tabla 1. Cantidad de prescripciones por receta médica

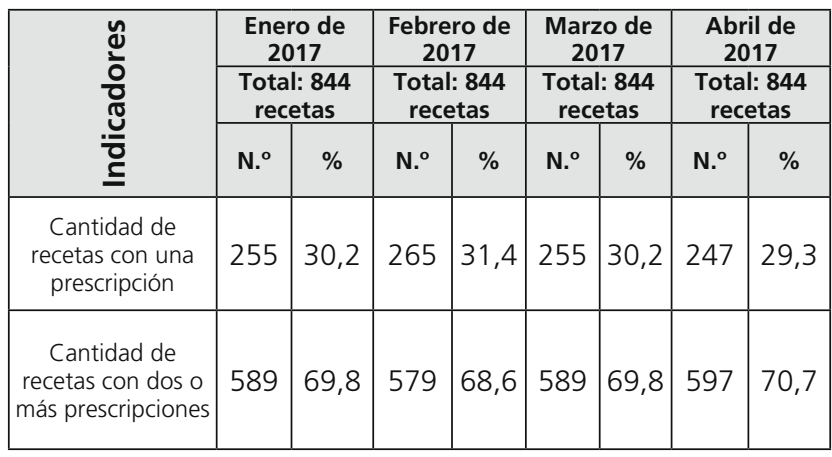

Fuente: Elaboración propia.

Las recetas médicas con mayor número de medicamentos en promedio seis a diez correspondían a los pacientes que consultaron en consultorios del Programa de Diabetes.

Teniendo en cuenta las recetas médicas con dos prescripciones, el $94 \%$ presentaban el nombre genérico del medicamento (tabla 2). El mes con un mayor porcentaje en cuanto a prescripción por nombre genérico fue abril con un 96,1\%. La utilización del nombre genérico de los medicamentos es un requisito del Ministerio de Salud Pública y Bienestar Social, el cual se encuentra establecido en la Ley 2320 de 2003 (14), ya que facilita el acceso a los medicamentos y también la adherencia del tratamiento, puesto que el nombre comercial puede generar confusiones al personal de la farmacia y también al paciente, pudiendo ocasionar fallos en la farmacoterapia.

Tabla 2. Porcentajes en general de cumplimiento o no de los indicadores de la OMS. considerando las recetas médicas con dos prescripciones.

\begin{tabular}{|c|c|c|c|c|}
\hline \multirow{2}{*}{ Indicadores } & \multicolumn{3}{|c|}{$\begin{array}{c}\text { Total de recetas: 1290 } \\
\text { (considerando las } \\
\text { recetas médicas con dos } \\
\text { prescripciones) }\end{array}$} \\
\cline { 2 - 5 } & \multicolumn{2}{|c|}{ Sí } & \multicolumn{2}{|c|}{ No } \\
\cline { 2 - 5 } & $\mathbf{N .}^{\mathbf{0}}$ & $\%$ & $\mathbf{N .}^{\circ}$ & $\%$ \\
\hline $\begin{array}{c}\text { Prescripciones de medicamentos } \\
\text { por nombre genérico }\end{array}$ & 1213 & 94 & 77 & 6 \\
\hline $\begin{array}{c}\text { Prescripciones de medicamentos } \\
\text { del listado de medicamentos } \\
\text { esenciales }\end{array}$ & 1285 & 99,6 & 5 & 0,4 \\
\hline $\begin{array}{c}\text { Prescripciones de medicamentos } \\
\text { clavedisponibles en la farmacia }\end{array}$ & 1119 & 86,7 & 171 & 13,3 \\
\hline
\end{tabular}

Fuente: Elaboración propia.

El 99,6\% de los prescriptores recetaron medicamentos del Listado de Medicamentos Esenciales (LME), dato que se elevó al 100\% durante el mes de enero. En los demás meses, se prescribieron medicamentos como enterogermina cápsulas, mupirocina crema y racecadotrilo cápsulas, los cuales no se encuentran en el LME.

Además, se observó que un 13,3\% de las recetas tenían prescripción de medicamentos sin existencia en la farmacia. Los medicamentos mayormente recetados que se encontraron en parte sin existencia fueron ibuprofeno suspensión oral, losartán comprimidos, amoxicilina y amoxicilina + sulbactam comprimidos.

En cuanto a las recetas, el $64,5 \%$ no cumplía con todos los requisitos legales. Se observó un alto nivel de cumplimiento en identificación del paciente (100\%), firma y sello del médico (99,7\%), sello de la institución (97,5\%) y prescripción según la especialidad del médico $(98,1 \%)$ (tabla 3).

El 92,3\% de las prescripciones contaba con el diagnóstico del paciente, el $79 \%$ tenía detallada la forma farmacéutica y el $53 \%$ la dosis (tabla 3 ). 
Tabla 3. Porcentaje de cumplimiento de los indicadores de la OMS analizados en las recetas médicas de los meses de enero a abril de 2017

\begin{tabular}{|c|c|c|c|c|}
\hline \multirow{3}{*}{ Indicadores } & \multicolumn{4}{|c|}{ Total de recetas: 3376} \\
\hline & \multicolumn{2}{|c|}{ Sí } & \multicolumn{2}{|c|}{ No } \\
\hline & N. ${ }^{\circ}$ & $\%$ & N. ${ }^{\circ}$ & $\%$ \\
\hline $\begin{array}{l}\text { Cumple con los } \\
\text { requisitos legales }\end{array}$ & 1199 & 35,5 & 2177 & 64,5 \\
\hline Letra legible & 2507 & 74,3 & 869 & 25,7 \\
\hline $\begin{array}{l}\text { Identificación del } \\
\text { paciente }\end{array}$ & 3375 & 100 & 1 & 0 \\
\hline $\begin{array}{l}\text { Número de cédula de } \\
\text { identidad }\end{array}$ & 1801 & 53,3 & 1575 & 46,7 \\
\hline $\begin{array}{l}\text { Cédula de identidad } \\
\text { completado por otro }\end{array}$ & 1474 & 43,7 & 1902 & 56,3 \\
\hline Diagnóstico & 3117 & 92,3 & 259 & 7,7 \\
\hline Dosis & 1788 & 53 & 1588 & 47 \\
\hline Firma y sello del médico & 3366 & 99,7 & 10 & 0,3 \\
\hline Sello de la institución & 3290 & 97,5 & 86 & 2,5 \\
\hline $\begin{array}{c}\text { Forma farmacéutica del } \\
\text { medicamento }\end{array}$ & 2672 & 79,1 & 704 & 20,9 \\
\hline Recetas con antibióticos & 968 & 28,7 & 2408 & 71,3 \\
\hline $\begin{array}{c}\text { Recetas con forma } \\
\text { farmacéutica inyectable }\end{array}$ & 15 & 0,4 & 3361 & 99,6 \\
\hline $\begin{array}{l}\text { Médico apto para } \\
\text { prescripción según } \\
\text { especialidad }\end{array}$ & 3311 & 98,1 & 65 & 1,9 \\
\hline
\end{tabular}

Fuente: Elaboración propia.

En la tabla 4, se observa el detalle del cumplimiento de indicadores detallados, de modo que es enero el mes en el que se registró en general un mayor cumplimiento.
Tabla 4. Comparativo de los indicadores de la OMS analizados en las recetas médicas de los meses de enero a abril de 2017

\begin{tabular}{|c|c|c|c|c|}
\hline \multirow[b]{2}{*}{ Indicadores } & $\begin{array}{l}\text { Enero } \\
\text { de } 2017\end{array}$ & $\begin{array}{l}\text { Febrero } \\
\text { de } 2017\end{array}$ & $\begin{array}{l}\text { Marzo } \\
\text { de } 2017\end{array}$ & $\begin{array}{c}\text { Abril de } \\
2017\end{array}$ \\
\hline & \begin{tabular}{|c|} 
Total de \\
recetas: \\
844 \\
\end{tabular} & \begin{tabular}{|c|} 
Total de \\
recetas: \\
844 \\
\end{tabular} & \begin{tabular}{|c|} 
Total de \\
recetas: \\
844 \\
\end{tabular} & $\begin{array}{l}\text { Total de } \\
\text { recetas: } \\
844 \\
\end{array}$ \\
\hline $\begin{array}{l}\text { Cumple con los } \\
\text { requisitos legales }\end{array}$ & $\begin{array}{l}50 \% \\
(422)\end{array}$ & $\begin{array}{c}37,6 \% \\
(317)\end{array}$ & $\begin{array}{c}27,6 \% \\
(233)\end{array}$ & $\begin{array}{c}27,1 \% \\
(227)\end{array}$ \\
\hline Letra legible & $\begin{array}{c}67,8 \% \\
(572)\end{array}$ & $\begin{array}{c}76,5 \% \\
(646)\end{array}$ & $\begin{array}{c}75,6 \% \\
(638)\end{array}$ & $\begin{array}{l}76 \% \\
(651)\end{array}$ \\
\hline $\begin{array}{l}\text { Identificación del } \\
\text { paciente }\end{array}$ & $\begin{array}{l}100 \% \\
(844)\end{array}$ & $\begin{array}{c}99,9 \% \\
(843)\end{array}$ & $\begin{array}{l}100 \% \\
(844)\end{array}$ & $\begin{array}{l}100 \% \\
(844)\end{array}$ \\
\hline $\begin{array}{l}\text { Número de cédula } \\
\text { de identidad }\end{array}$ & $\begin{array}{c}58,4 \% \\
(493)\end{array}$ & $\begin{array}{c}57,5 \% \\
(485)\end{array}$ & $\begin{array}{l}48 \% \\
(405)\end{array}$ & $\begin{array}{c}48,8 \% \\
(418)\end{array}$ \\
\hline $\begin{array}{l}\text { Cédula de identidad } \\
\text { completado por otro }\end{array}$ & $\begin{array}{c}37,9 \% \\
(320)\end{array}$ & $\begin{array}{c}41,1 \% \\
(347)\end{array}$ & $\begin{array}{c}49,3 \% \\
(416)\end{array}$ & $\begin{array}{c}47,7 \% \\
(391)\end{array}$ \\
\hline Diagnóstico & $\begin{array}{l}92,1 \% \\
(777)\end{array}$ & $\begin{array}{c}92,7 \% \\
(782)\end{array}$ & $\begin{array}{c}93,2 \% \\
(787)\end{array}$ & $\begin{array}{l}93,1 \% \\
(771)\end{array}$ \\
\hline Dosis & $\begin{array}{c}49,8 \% \\
(420)\end{array}$ & $\begin{array}{c}57,9 \% \\
(489)\end{array}$ & $\begin{array}{l}52,7 \% \\
(445)\end{array}$ & $\begin{array}{c}52,1 \% \\
(434)\end{array}$ \\
\hline $\begin{array}{l}\text { Firma y sello del } \\
\text { médico }\end{array}$ & $\begin{array}{l}100 \% \\
(844)\end{array}$ & $\begin{array}{c}99,6 \% \\
(841)\end{array}$ & $\begin{array}{c}99,5 \% \\
(840)\end{array}$ & $\begin{array}{c}99,5 \% \\
(841)\end{array}$ \\
\hline Sello de la institución & $\begin{array}{c}96,4 \% \\
(814)\end{array}$ & $\begin{array}{c}96,8 \% \\
(817)\end{array}$ & $\begin{array}{c}98,6 \% \\
(832)\end{array}$ & $\begin{array}{c}98,6 \% \\
(827)\end{array}$ \\
\hline $\begin{array}{l}\text { Forma farmacéutica } \\
\text { del medicamento }\end{array}$ & $\begin{array}{c}84,1 \% \\
(710) \\
\end{array}$ & $\begin{array}{c}81,8 \% \\
(690)\end{array}$ & $\begin{array}{c}75,8 \% \\
(640) \\
\end{array}$ & $\begin{array}{c}75,6 \% \\
(632) \\
\end{array}$ \\
\hline $\begin{array}{l}\text { Recetas con } \\
\text { antibióticos }\end{array}$ & $\begin{array}{c}31,4 \% \\
(265)\end{array}$ & $\begin{array}{c}24,5 \% \\
(207)\end{array}$ & $\begin{array}{c}28,4 \% \\
(240)\end{array}$ & $\begin{array}{c}29,8 \% \\
(256)\end{array}$ \\
\hline $\begin{array}{l}\text { Recetas con forma } \\
\text { farmacéutica } \\
\text { inyectable }\end{array}$ & $\begin{array}{l}0,5 \% \\
(4)\end{array}$ & $\begin{array}{c}0,5 \% \\
(4)\end{array}$ & $\begin{array}{l}0,6 \% \\
(5)\end{array}$ & $\begin{array}{c}0,2 \% \\
(2)\end{array}$ \\
\hline $\begin{array}{l}\text { Médico apto para } \\
\text { prescripción según } \\
\text { especialidad }\end{array}$ & $\begin{array}{c}98,6 \% \\
(832)\end{array}$ & $\begin{array}{c}97,9 \% \\
(826)\end{array}$ & $\begin{array}{c}98,5 \% \\
(831)\end{array}$ & $\begin{array}{c}98,4 \% \\
(822)\end{array}$ \\
\hline
\end{tabular}

Fuente: Elaboración propia.

El 100\% de los errores se resolvió mediante comunicación oral entre el farmacéutico y otro profesional de la salud.

\section{Discusión}

De las recetas evaluadas en este estudio, el $64,5 \%$ presentaban al menos un tipo de error, cifra menor a lo reportado por otras investigaciones, que va desde el 82 al $90 \%(15,16)$. Esto es un porcentaje alto considerando que cada error puede suscitar una comprensión errónea de quienes tienen la responsabilidad de interpretar las recetas y dispensar los medicamentos incluidos en ellas. 
En esta investigación, un error frecuente fue el relacionado con la dosis del medicamento prescrito, lo que coincide con lo mencionado en la literatura, en que los principales errores en las recetas médicas eran los relacionados con la forma farmacéutica y dosis de los medicamentos. En nuestro estudio, estos errores alcanzaron el 20,9 y el $47 \%$, respectivamente, cifras diferentes presentadas por otros estudios $(8,16,17)$.

En las recetas con más de dos medicamentos prescritos, el riesgo de error aumenta 2,4 veces. Considerando que en este estudio se encontraron recetas con hasta diez prescripciones, preocupa el hecho de que los pacientes con muchos fármacos recetados, además de la probabilidad de presentar los problemas propios de una polimedicación (efectos adversos, interacciones, baja adherencia al tratamiento), se deban enfrentar a un mayor riesgo de recetas con errores (16).

El indicador de letra legible muestra un porcentaje de no cumplimiento del $25,7 \%$, por lo que es importante indicar que esto puede ocasionar errores graves que incluso podrían causar la muerte del paciente (18).

En varios estudios realizados para identificar los principales errores en la medicación, se encontró que la poca legibilidad en las prescripciones ocasiona fallos en el sistema que pueden llevar a la muerte $(18,19,20)$.

Otro estudio que hace referencia a este indicador es el realizado en el área de consulta de farmacia externa en un hospital de alta complejidad, en el cual el resultado obtenido es que, del $100 \%$ de recetas analizadas, el $10,9 \%$ presentaban letra ilegible (21).

La incorporación de sistemas de prescripciones electrónicas mejoraría no solo los errores asociados a legibilidad, sino también lo haría con aquellos asociados a la omisión de información, porque el mismo sistema obligaría al prescriptor a completar todos los datos. De esta manera, los sistemas de prescripción computarizados pueden potencialmente mejorar la calidad de los procesos de medicación $(5,12,22)$.

Los errores de medicación se consideran acontecimientos prevenibles, y si bien muchos de ellos no provocan daño (son leves o no alcanzan al paciente), hay un porcentaje que sí podría potencialmente causar un daño tanto a pacientes como familiares, además de los costos asistenciales y económicos que implicaría (4). Por ello, se sugieren medidas como mejoras en los sistemas de almacenamiento, incorporación de sistemas de dispensación automatizados, hasta métodos que aumenten la concentración y comunicación del personal de la farmacia. Si bien todas ellas contribuirian a la prevención de errores, lo más importante, y que debe estar presente, es el chequeo de todas las etapas, especialmente al final del proceso, por un profesional del área farmacéutica (6, 23, 24).

El farmacéutico, mediante las intervenciones farmacéuticas, ha ido demostrado a lo largo de los últimos años un papel en la mejora de resultados en la salud de los pacientes (25).

\section{Conclusión}

Se registró un bajo cumplimiento de recetas según los indicadores de la OMS. Esto evidencia que existe un problema que ha de ser corregido desde el inicio del acto de la prescripción, para evitar futuros errores que puedan alcanzar al paciente, si bien muchos de ellos son leves o no alcanzan al paciente, hay un porcentaje que sí podría potencialmente causar un daño a los pacientes, además de los costos asistenciales y económicos que implicaría.

\section{Referencias}

1. Kohn L. Why do errors happen? En: Kohn LT, Corrigan JM, Donaldson MS, editor, Committee on Quality of Health Care in America, To Err is Human: building a safer health system. Washington D.C.: National Academy Press; 2000. p. 49-68.

2. Adubofour KO, Keenan CR, Daftary A, et al. Strategies to reduce medication errors in ambulatory practice. $\mathrm{J}$ Natl Med Assoc 2004;96(12):1558-64.

3. Otero López, MJ. El papel del farmacéutico en la gestión de la seguridad de los medicamentos diez años después de la publicación del informe "Errar es humano". Farmacia Hospitalaria. 2010;34(4):159-162.

4. Otero López, MJ. La gestión de riesgos en la prevención de los errores de medicación. En: Sociedad Española de Farmacia Hospitalaria, Curso de formación continuada en farmacoterapia de la SEFH. Madrid: Aran; 2007. p 111147.

5. Aronson JK. Medications errors: definitions and classification. Br J Clin Pharmacol. 2009; 67(6):599-604.

6. Cheung KC, Bouvy ML, De Smet PA. Medications errors: the importance of safe dispensing. Br J Clin Pharmacol. 2009;67(6):676-80.

7. Knudsen P, Herborg H, Mortensen AR, et al. Preventing medication errors in community pharmacy: frequency and seriousness of medication errors. Qual Saf Health Care. 2007;16(4):291-296. 
8. Pastó-Cardona L, Masuet-Aumatell C, Bara-Oliván B, et al. Estudio de incidencia de los errores de medicación en los procesos de utilización del medicamento: prescripción, trascripción, validación, preparación, dispensación y administración en el ámbito hospitalario. Farmacia Hospitalaria. 2009;33(5):257-268.

9. Berdot S, Sabatier B, Guillaizeau F, et al. Evaluation of drug administration errors in a teaching hospital. BMC Health Serv Res. 2012;12(60): 1-8.

10. Dos Santos L, Winkler N, Dos Santos M, et al. Description of medication errors detected at a drug information centre in Southern Brazil. Pharmacy Practice. 2015;13(1):524.

11. Tully MP. Prescribing errors in hospital practice. Br J Clin Pharmacol. 2012;74(4):668-75.

12. Keers RN, Williams SD, Vattakatuchery JJ, et al. Prevalence, nature and predictors of prescribing errors in mental health hospitals: a prospective multicentre study. BMJ Open. 4(9):e006084.

13. Ryan C, Ross S, Davey P, et al. Prevalence and causes of prescribing errors: the PRescribing Outcomes for Trainee Doctors Engaged in Clinical Training (PROTECT) study. PloS one. 2014;9(1):e79802.

14. Paraguay, Congreso de la Nación Paraguaya, Ley 2320, promoción de la utilización de medicamentos por su nombre genérico (3 dic 2003).

15. Lee BH, Lehmann CU, Jackson EV, et al. Assessing controlled substance prescribing errors in a pediatric teaching hospital: an analysis of the safety of analgesic prescription practice in the transition from the hospital to home. The Journal of Pain. 2009;10(2):160-166.

16. Graciano Silva, M das D, Borges Rosa, M, Dean Franklin, $\mathrm{B}$, et al. Concomitant prescribing and dispensing errors at a Brazilian hospital: a descriptive study. Clinics. 2011;66(10):1691-1697.
17. Díaz-Carrasco MS, Pareja A, Yachachi A, et al. Prescription errors in chemotherapy. Farmacia Hospitalaria. 2007;31(3):161-164.

18. Velo GP, Minuz P. Medication errors: prescribing faults and prescription errors. Br J Clin Pharmacol. 2009;67(6):624628.

19. Otero MJ, Martín R, Robles D, et al. Errores de medicación. En: Bonal Falgas J, Dominguez-Gil H, Gamundi Planas MC, editor. Farmacia hospitalaria. Madrid: SCM SL (Doyma);2002. p. 713-47.

20. Bates K, Beddy D, Whirisky C, et al. Determining the frequency of prescription errors in an Irish hospital. Ir J Med Sci. 2010;179(2):183-186.

21. Pinedo Y, Romero JV, Merino FN. Cumplimiento de buenas prácticas de prescripción en pacientes hospitalizados. Interciencia RCCI. 2014;5(1):26-30.

22. Chen YF, Neil KE, Avery AJ, et al. Prescribing error and other problems reported by community pharmacists. Ther Clin Risk Manag. 2005;1(4):333-342.

23. Anacleto TA, Perini E, Rosa MB, et al. Drug-dispensing errors in the hospital pharmacy. Clinics. 2007;62(3):243-50.

24. Beso A, Franklin BD, Barber N. The frequency and potential causes of dispensing errors in a hospital pharmacy. Pharm World Sci. 2005; 27(3):182-90.

25. Maidana GM, Lugo GB, Vera Z, Pérez S, Mastroianni PC. Evaluación de un programa de atención farmacéutica en pacientes con diabetes mellitus tipo 2. Pharm Care Esp. 2016; 18(1): 3-15. 